$$
7 .
$$$$
8
$$$$
9
$$$$
10
$$$$
11
$$

\title{
The openCARP Simulation Environment for Cardiac Electrophysiology
}

\author{
Gernot Plank $^{1, *, \#}$, Axel Loewe ${ }^{2, *, \#}$, Aurel Neic ${ }^{3, *, \#}$, Christoph Augustin ${ }^{1}$, \\ Yung-Lin Huang ${ }^{4,5}$, Matthias A. F. Gsell ${ }^{1}$, Elias Karabelas ${ }^{6}$, Mark Nothstein ${ }^{2}$, \\ Anton J. Prass1 ${ }^{1}$, Jorge Sánchez ${ }^{2}$, Gunnar Seemann ${ }^{4,5, * \#}$, Edward J. Vigmond ${ }^{7, *, \#}$ \\ ${ }^{1}$ Gottfried Schatz Research Center, Division of Biophysics, Medical University of Graz, Graz, Austria \\ ${ }^{2}$ Institute of Biomedical Engineering, Karlsruhe Institute of Technology (KIT), Karlsruhe, Germany \\ ${ }^{4}$ Institute for Experimental Cardiovascular Medicine, University Heart Center Freiburg · Bad Krozingen, Medical \\ Center - University of Freiburg, Freiburg, Germany \\ ${ }^{5}$ Faculty of Medicine, University of Freiburg, Freiburg, Germany \\ ${ }^{6}$ Institute of Mathematics and Scientific Computing, University of Graz, Graz, Austria \\ ${ }^{7}$ Liryc Cardiac Modeling Institute, Fondation Bordeaux University, Bordeaux, France \\ *Corresponding authors: info@opencarp.org \\ "These authors contributed equally to this work.
}

${ }^{3}$ NumeriCor $\mathrm{GmbH}$, Graz, Austria

\begin{abstract}
Background and Objective: Cardiac electrophysiology is a medical specialty with a long and rich tradition of computational modeling. Nevertheless, no community standard for cardiac electrophysiology simulation software has evolved yet. Here, we present the openCARP simulation environment as one solution that could foster the needs of large parts of this community.

Methods and Results: openCARP and the Python-based carputils framework allow developing and sharing simulation pipelines which automate in silico experiments including all modeling and simulation steps to increase reproducibility and productivity. The continuously expanding openCARP user community is supported by tailored infrastructure. Documentation and training material facilitate access to this complementary research tool for new users. After a brief historic review, this paper summarizes requirements for a high-usability electrophysiology simulator and describes how openCARP fulfills them. We introduce the openCARP modeling workflow in a multi-scale example of atrial fibrillation simulations on single cell, tissue, organ and body level and finally outline future development potential.

Conclusion: As an open simulator, openCARP can advance the computational cardiac electrophysiology field by making state-of-the-art simulations accessible. In combination with the carputils framework, it offers a tailored software solution for the scientific community and contributes towards increasing use, transparency, standardization and reproducibility of in silico experiments.
\end{abstract}

\section{Introduction}

Computational modeling and simulation of cardiac electrophysiology (CEP) has emerged in the last decades and is now playing a pivotal role in basic cardiology research [1]. It also shows high promise as a clinical research tool, for device and drug development [2] and even as a complementary clinical modality, aiding in diagnosis, therapy stratification and planning in future precision cardiology [3]. A key motivation driving CEP model development is the unique ability of providing a mechanistic framework for integrating disparate experimental or clinical data gathered in in vivo, in vitro or ex vivo and subjecting these to thorough quantitative analysis in a matching in silico setting. Such in silico CEP models allow study of complex cause-effect relationships at a level of quantitative accuracy and biophysical detail beyond what is feasible today with any other research modality. 
bioRxiv preprint doi: https://doi.org/10.1101/2021.03.01.433036; this version posted June 10, 2021. The copyright holder for this preprint (which was not certified by peer review) is the author/funder, who has granted bioRxiv a license to display the preprint in perpetuity. It is made available under aCC-BY 4.0 International license.

Advanced in silico CEP models facilitate the observation of almost any quantity of interest at high spatio-temporal resolutions at scales ranging from cellular to organ, including studies on human hearts, without being limited by ethical constraints. These advantages have led to a marked increase in modeling-based or modeling-augmented publications in cardiology since the early 2000s (Figure 1) with a wide variety of different software implementations, e.g. illustrated in [4-8] or a joint verification benchmark effort of the CEP community [9].

Today, simulations of cardiac function in anatomically accurate and biophysically detailed in silico models have become feasible. Key factors hampering a further adoption of in silico CEP models in advanced application scenarios in basic research, industrial device development and clinical decision making are the limited access to cutting edge simulation technology, and the inherent complexities involved in using such sophisticated tools. These factors render setting up and performing advanced simulation studies a challenging endeavor. In this paper, we present openCARP, a CEP modeling environment fully open for academic use that aims to lift these limitations to increase accessibility and, thus, boost adoption of in silico analysis of CEP. To cope with the broad range of demands in terms of efficiency, flexibility and usability, the openCARP modeling environment comprises two major software components: the actual openCARP simulator and an open source Python framework, referred to as carputils, for describing in silico experiments. carputils facilitates building and sharing multiscale workflows by standardization of parameterization, execution, and archiving of simulations, to increase reproducibility and enhance robustness and reliability of complex CEP studies. In general, carputils can be adapted to support other CEP simulators as well. Infrastructure supporting interaction combined with extensive tailored documentation and regular user meetings provide the basis for fostering a vibrant user community.

This paper first gives a brief historic overview of the field of CEP simulation software, from the early pioneering days to the current situation that motivated the development of the openCARP simulator and carputils. Then, we review requirements for a CEP simulation environment to accommodate the needs of a wide user base. We introduce the openCARP CEP simulator and the carputils Python framework, together with pre- and post-processing components. The use of these tools is described along a typical workflow for setting up a state-of-the-art in silico simulation study, that spans from single cell to organ level, covering a wide range of user needs in the CEP modeling and simulation field.

\subsection{Historical CEP Software Development}

It was not before the mid-1980s that computational models for studying bioelectrical activity at the tissue level emerged [10,11]. Unlike modeling work focused on vascular mechanics or hemodynamics, for which - owing to close similarities to applications of industrial relevance - commercial software became available early on, this was not the case for CEP modeling. In the absence of such software, academia had to develop CEP modeling solutions, which has remained that way up until today. While commercially available multiphysics simulators may have CEP modules, they are limited in terms of speed and capability [12]. In general, these do not meet the demands of state-of-the-art CEP studies

which have undergone a marked transformation over the past decade, leading to a dramatic increase in complexity $[13,14]$.

As a consequence, the vast majority of published CEP modeling studies have relied on academic in-house codes [15-23]. These software packages have been largely developed by individual laboratories as side projects in support of their applied research work, typically focused on understanding mechanisms underlying the formation and maintenance of arrhythmias [24, 25] and their therapies [26-28]. The direction of software development

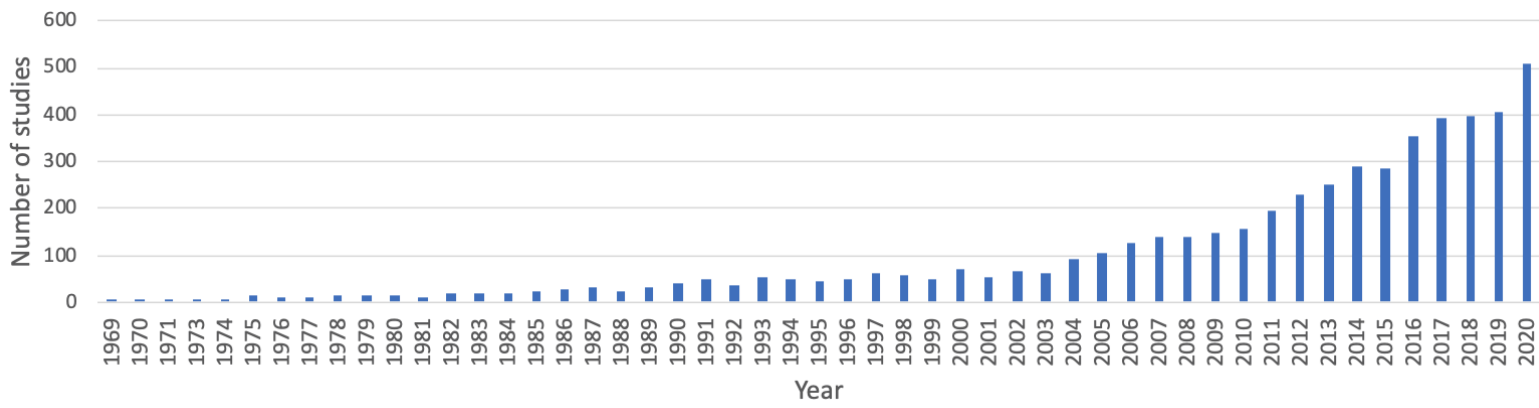

Figure 1: PubMed-listed studies with "(atria* OR ventric* OR cardi*) AND ("computer model" OR "mathematical model" OR "computational model" OR "in silico")" in title or abstract. 

made available under aCC-BY 4.0 International license.

has been largely steered by the needs of funded projects. Often, this led to ad-hoc development processes yielding research prototypes but no sustainable software products for long-term use, rather than a roadmap-based development targeting longer term strategic goals. Moreover, particularly during the pioneering years, the modeling infrastructure encoded the specific expertise of individual labs. Sharing of software or models across labs was uncommon. Research on CEP resulting in discovery has been traditionally regarded as being of higher academic merit than research on building the enabling CEP modeling methodology, which requires a similar amount of human resources. Thus, modeling has been significantly less well funded, which has opened a gap between the often highly ambitious scientific goals and the available technical capabilities [29].

For at least the first three decades of CEP modeling, the single lab paradigm was the prevailing approach in the development of CEP modeling infrastructure. An important factor rendering this approach viable was the simple nature of CEP simulations during these early years, making it feasible for a single graduate student to develop and write code for the problem at hand. Models of cellular dynamics were relatively simple [30-32], and geometric representations were anatomical abstractions, comprising 1D strands, 2D sheets or, later in the late nineties when computational resources became more powerful, 3D slab or wedge preparations. These regular domains lent themselves quite naturally to simpler spatial discretization techniques, with the finite difference method (FDM) appropriate for the vast majority of studies. Numerical techniques were less well developed and much simpler to implement, or generic implementations made available as numerical libraries were integrated.

\subsection{Current State of CEP Modeling}

Over the past decade though, the complexity of CEP simulations has increased exponentially as the result of several factors:

i) faster, and higher resolution tomographic imaging modalities

ii) an explosion of biological knowledge leading to more components in cell models, as well as more complicated formal descriptions of these components and interactions between them

iii) more elaborate numerical methods which improve performance at the cost of increased complexity

iv) evolving computer architectures which demand specific data layouts and algorithms to fully exploit the resources

Complexity has increased beyond the capabilities of a single lab development paradigm.

State-of-the-art modeling studies use unstructured, high resolution, image-based tomographic reconstructions to reflect individual cardiac anatomies with high geometric fidelity and avoid spurious boundary artefacts introduced by jagged surfaces of Cartesian grids. Unstructured tesselation has prompted more sophisticated discretization techniques, such as the finite element method (FEM) [33] or the finite volume method (FVM) [34], which are substantially more costly to implement and time-consuming to develop a profound understanding of. CEP is studied at the organ scale in biventricular or biatrial models and recently models representing all chambers of the heart are beginning to be used [35].

Such four chamber models account for anisotropic tissue properties and discrete conduction pathways in all chambers, the specialized cardiac conduction system comprising sinus node [36-38], atrio-ventricular node [39] and the His-Purkinje system [40]. Pathologies altering conduction velocities or pathways such as ischemia [41], infarct scars and surrounding transitional tissue [42], or various types of fibrotic lesions [43-45] are considered at increasing levels of physiological [46] and structural detail [47-50].

Along with the structural heterogeneity, a large number of cellular models are needed to account for functional heterogeneity across different regions of the heart, often with subtle variations within regions to account for gradients in protein expression that alter action potential (AP) morphology. The CellML markup language, along with its repository (cellml.org), was created to aid in the standardization and dissemination of these models [51]. Beyond the numerous issues in developing the software, major challenges in building in silico experiments have to be addressed. Sophisticated workflows have been conceived to facilitate automated translation of segmented image data sets into discrete meshes suitable for simulations [52-54]. These workflows are constantly refined to render the production of increasingly larger numbers of individualized models, often referred to as virtual cohorts, feasible [35]. In the process of functional personalization of such models, tuning parameters have to be identified to achieve a close fit with observed data. Experimental or clinical protocols are mimicked in silico to match in vivo experiments or clinical conditions ever more closely. These procedures typically require simulating prolonged periods to ensure convergence to a limit cycle, and the number of simulations executed within optimization loops tend to be high. Overall, these factors, in combination with the numerical demands imposed by CEP - the fast upstroke of the action potential translates into depolarization wave fronts of limited spatial extent $(<1 \mathrm{~mm})-$ render the execution of modeling studies a challenging endeavour. Such studies can only be executed with highly efficient, 
robust and versatile CEP simulation tools. The costs in terms of personnel necessary to develop such tools from scratch is daunting. For instance, the cost of development of CARP, the proprietary predecessor of openCARP, is estimated to be around 50 person-years, not including any of the pre- and post-processing codes necessary for building the workflows.

\subsection{Towards a Common Software}

In other fields, certain software has been adopted by a critical mass of the community and thus have become the de facto standard for the entire field. Examples of this are Neuron for neural simulation [55], and GAMESS for chemistry [56]. While the need for standardized, open software has been recognized before within the CEP modeling community, no software has achieved such a status. Preceding initiatives in this vein include openCMISS [18] and Chaste [57], but their success has been mixed so far, as their rate of adoption has been rather marginal. Reasons are multifactorial: For groups developing numerical methods, there is limited demand as they have the capability to build custom tailored frameworks themselves [23,58-62] that facilitate the implementation of disruptive changes at any time, without the frictional losses involved in community projects where changes have to be agreed upon by various stakeholders. This is in stark contrast to the demands within the applied CEP modeling community. A broad web-based survey conducted by us in 2017 showed that for a convergence in the CEP field, simulation environments are needed which meet most or, ideally, all of the following requirements:

- Feature completeness, i.e. a simulator should have the features to be able to replicate a large share of published modeling studies using other software [57,63]. It must be able to perform the functions of any software it is replacing, as well as offer new functionality.

- The simulator needs to be made available under a license that allows unrestricted academic use [64].

- A streamlined installation process for all popular deployment targets without the need to deal with intricate technical challenges of compilation and software dependencies [64].

- An intuitive and flexible user interface that exposes input parameters in an intuitive way while accommodating a wide range of experimental conditions and protocols. Consistent interfaces are the basis for a positive and intuitive user experience [65].

- Comprehensive user documentation for all tools, combined with extensive training material, such as tutorials for all simulator features to get new users started within a reasonable time, and a tractable learning curve [64].

- The simulation environment should provide streamlined, standardized workflows to share, reproduce, and archive in silico experiments [66]. It should be easy to export results, together with all relevant input data, in one bundle. A high degree of standardization also facilitates the sharing of expertise between groups.

- A vibrant user and developer community is key for a sustainable research software [67]. These communities should be supported by interactive platforms, spur development (feature requests, bug reports), and interact via webcasts and user meetings [64].

- The simulator should be computationally efficient on all common hardware platforms ranging from local workstations to national high performance computing facilities [68].

- Code documentation with a thorough description of classes, methods and interfaces, as well as the overall software architecture, lowers the barrier for users becoming developers and contributing to the project. This documentation, in combination with a modular and extensible architecture, is the basis for sustainable research software [69].

- Import from (e.g. CellML [51] for cellular models) and export to (e.g. VTK [70]) common data formats increases interoperability. Easy integration in existing pre- and post-processing workflows attracts a wider range of users. The version of the simulator code used to generate a result should be clearly identified and accessible via a persistent and citable identifier.

- A high level of trust in the correctness of the software implementation is instrumental. The functionality of the simulator needs to be verified and constantly controlled by quality assurance measures. Before entering the master branch, new code should be reviewed by maintainers. Test cases should cover all use cases and be executed for each build before deployment [64]. The necessary trust is best established in a positive feedback loop wherein a critical mass of CEP modeling labs adopts and successfully uses the software for a broad range of modeling applications over prolonged periods. 


\section{Methods and Results}

The main objective of the openCARP initiative is to provide and establish simulation software within the CEP modeling community which fulfills the criteria outlined above. To better meet these user requirements, the openCARP modeling environment is organized as comprising two major software bundles, the actual code for executing simulations, i.e., the openCARP simulator, and a framework for describing in silico experiments, referred to as carputils (Fig. 2). The openCARP simulator has been developed with the following objectives in mind:

- Accessibility: The main distribution mechanism of openCARP emphasizes binary packaging for commonly used platforms (currently Linux and macOS), platform-independent Docker containers, and a detailed documentation supporting source installations on all levels of computers including large-scale national HPCs. Only providing a source installation excludes major parts of the user community who are not sufficiently versed in compiling scientific software, or do not want to manage a complex software stack, especially given the release requirements and potential conflicts with compilers and underlying software libraries. The source code of all openCARP components is maintained in the public GitLab instance git.opencarp.org. carputils is published under the Apache 2.0 open source license, and the openCARP simulator under the Academic Public License restricting the open use to academic non-profit cases. A commercial license can be requested.

- Interoperability: openCARP builds on two decades of CEP modeling experience gained with its proprietary predecessors, CARP [16] and acCELLerate [17], with a stable user base about 130 registered users. The predecessor software packages have been extensively used by several CEP modeling groups and led to $>250$ peer-reviewed journal publications, covering the full range of modeling issues including numerical methods [71], anatomical modeling and model functionalization [48,72] as well as a broad range of applications such as formation and maintenance of arrhythmias [73,74], EP therapies [75,76], diagnostic [77, $78]$ and therapeutic applications $[79,80]$. The openCARP simulator has been built from scratch in terms of code, but the user interface is consistent with previous proprietary versions of $C A R P$ [16] to facilitate re-use of a large number of existing experiments. Moreover, openCARP interoperates with relevant community standards by providing input and output interfaces VTK [70] and CellML [51], for example, either directly or via other tools. All openCARP specific formats and standards are openly available and concisely described, with adequate tools for their management provided as source code.

- Performance: openCARP has been implemented from scratch in C++ (2011 standard) but follows the same discretization and solver schemes that have been used successfully in the predecessor code CARP [16,71]. Briefly, the openCARP simulator spatially discretizes the partial differential equations underlying the monoor bidomain model (or potentially other physics like mechanics) using the FEM [81, 82] with linear basis functions. The bidomain equations are cast in the elliptic-parabolic form and decoupled to be solved sequentially [83], with various time stepping options including fully explicit Euler or $\theta$-schemes, with or without operator splitting, leading to fully explicit or implicit-explicit solver schemes as the reaction term is always treated explicitly. The interested reader is referred to the openCARP user manual for numerical details. The FEM implementation makes use of parallel mesh management and mesh partitioning [68]. The resulting linear systems of equations are solved with PETSC [84], with various pre-configured solver options [85-87], under exploitation of parallel algebraic matrix-vector operations, parallel I/O, and timing routines among others. On each node, the embarassingly parallel LIMPET library [88] is used to calculate the cellular electrophysiology models. Time integration schemes for these ODE systems can be controlled per variable and include Runge-Kutta, Rush-Larsen, Rosenbrock [89] and advanced schemes accessible via CVODE [90] with adaptive step size and error control. While profiling and benchmarking plays a central role during development, openCARP does not yet employ detailed benchmarking or profiling during integration testing. Currently, integration tests are binned into different execution time groups and tests changing group are marked as failed.

- Transparency: The availability as source code will benefit the technically affine model developers in the CEP modeling community by allowing implementation of additional features, the critical revision of numerical schemes, and the identification of weaknesses. This will trigger constant re-engineering of the software and, thus, help improve software quality. The software is version controlled using git and connected to continuous integration / continuous delivery (CI/CD) services with integration testing of most simulation setups (currently 74 individual simulations). We have opted against unit testing at the current stage of development. Release versions of openCARP [91] are automatically archived and uploaded to the RADAR4KIT repository as part of the continuous deployment pipelines [92]. Submission information packages are built in BagIt format and comprise the source code, the binary versions for the support platforms, the respective version of the documentation and test reports. Metadata is automatically extracted 

made available under aCC-BY 4.0 International license.

from machine-readable files in the git repositories and provided according to the DataCite 4.3 scheme [93]. A DOI is minted for all published and archived versions to ensure long-term accessibility.

carputils is a framework designed to define and execute in silico experiments by encoding the complex workflows of advanced CEP simulation studies in a reasonably standardized manner. By providing an additional abstraction layer on top of the openCARP simulator itself, we aim to achieve the following objectives:

- Usability: The main focus of carputils is on providing a modeling and simulation environment which enables researchers to carry out a wide range of studies out of the box. Only a physiological understanding of the problem at hand should be necessary for executing such experiments, not knowledge of underlying numerical details.

- Reducing complexity: In silico experiments defined with carputils facilitate learning by exposing only a small number of relevant parameters which are needed for controlling a given experiment.

- Reproducibilty: By providing mechanisms for easy sharing of in silico experiments, carputils contributes to the concept of Open Science and facilitates reproducibility and reuse. Owing to the complexity of experiments, the ability to share them has been severely hampered, even within the same laboratory, let alone across different ones. We overcome barriers posed by numerous differences in local installations, inconsistencies between code revisions, and the management of high dimensional input parameter spaces defining a setup: meshes, label fields, electrode definitions and pacing protocols, limit cycle state vectors, structural entities such as fibrotic lesions or scars, functional heterogeneities, conduction system topology, and parabolic solver method, to name but a few important ones.

- Productivity: carputils aims to boost productivity by providing standardized workflows for common tasks such as setting conduction velocities, computing limit cycles of ionic models to generate initial states, interrogate restitution properties, or post-processing and visualization of outputs.

- Abstraction: carputils uncouples technicalities of executing simulations on different platforms from experiment design such that they can be executed on any supported platform - from laptops to national HPC facilities - using the exact same command line. Platform-specific aspects related to scheduling and launching of jobs are hidden from the user, and encapsulated in abstract platform descriptions. Only generic user choices such as the number of processes to be used in a simulation must be provided.

- Quality assurance: Support for regression testing of all major simulator features is built in, including automated nightly building and testing of the entire software stack. carputils encodes a range of verification benchmarks such as the CEP N-version benchmark [9] and a suite of additional performance benchmarks of varying level of complexity, ranging from biventricular slices up to whole heart models, for measuring simulator performance, or for investigating the effect of numerical settings.

Additional open source software components have been developed to support the modeling, simulation and visualization when using openCARP as shown in Fig. 2. meshtool [54] (License: GPL v3) allows generating or interacting with geometric data. carputilsGUI (License: Apache 2.0) is a web-based graphical user interface to control specific carputils experiments and visualize results within the browser for low-threshold entry to openCARP. meshalyzer (License: GPL v3) is a tailored visualization program for CEP, even though VTK export allows seamless visualization in ParaView [94] as well.

openCARP provides documentation for users with different levels of experience. Video tutorials introduce users to the basics of CEP modelling and guide them through their first steps with openCARP. Further videos introduce standard simulation pipelines and progressively advanced features covering the whole gamut from single cell simulations to 3D heart models including pre- and post-processing steps using the carputils framework. carputils examples cover common simulation scenarios and provide a starting point to develop custom carputils experiments in combination with the API documentation. The openCARP reference documentation contains all openCARP parameters to give full control of all aspects. Continuous deployment pipelines ensure that documentation (web page, PDF) is always in sync with the code by automatically generating these artifacts based on the code.

\subsection{Workflows and Use Cases}

In the following sections, we introduce the openCARP simulation framework by covering common workflows for use cases ranging from the cellular to the organ level. Specifically, we elucidate the key processing steps to implement a human biatrial model of persistent atrial fibrillation. An overview of a standardized processing workflow is given in Fig. 3. 
bioRxiv preprint doi: https://doi.org/10.1101/2021.03.01.433036; this version posted June 10, 2021. The copyright holder for this preprint (which was not certified by peer review) is the author/funder, who has granted bioRxiv a license to display the preprint in perpetuity. It is made available under aCC-BY 4.0 International license.

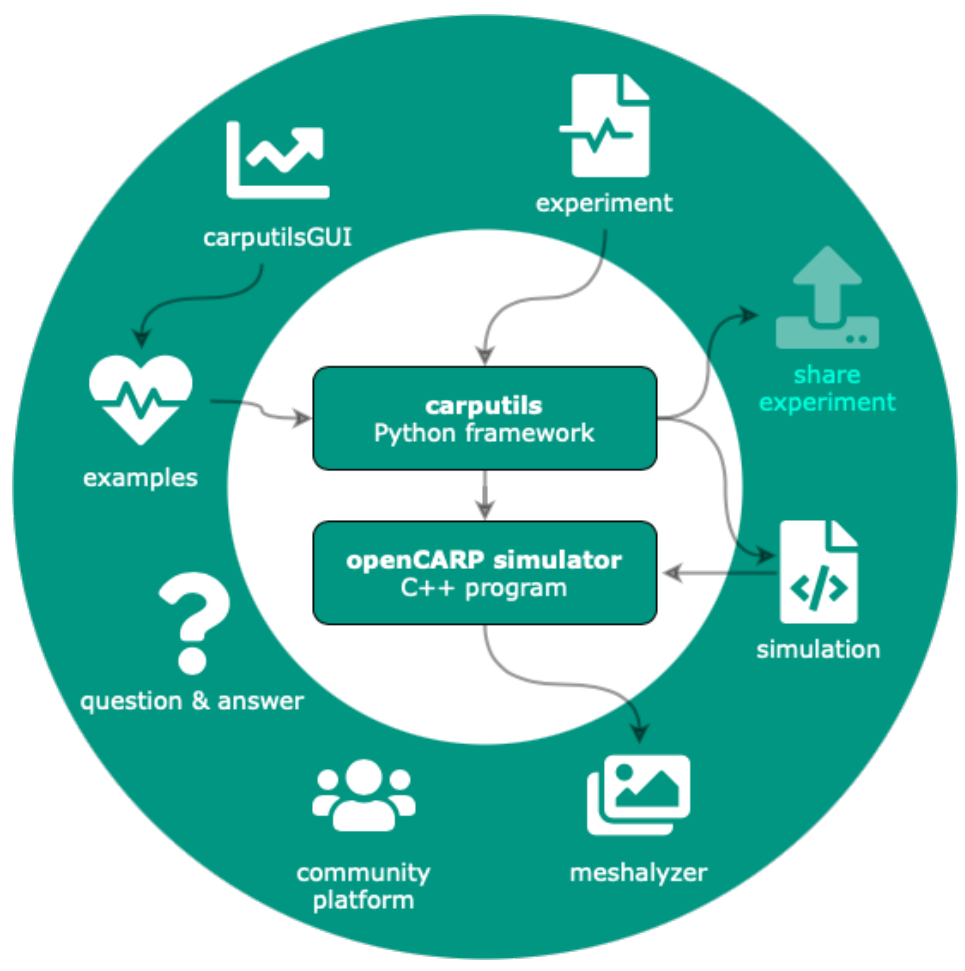

Figure 2: The openCARP ecosystem comprises several components that interact with its central pillars: carputils and the openCARP simulator. Users can write their own experiments using the carputils Python framework, or start with existing examples. carputils will set up the simulation, launch the openCARP simulator, perform postprocessing steps and call meshalyzer for visualization. Apart from the commandline interface, examples can also be parametrized, run and visualized using the web-based carputilsGUI. A community platform including a question \& answer system completes the openCARP ecosystem.

\subsubsection{Cellular Level}

The ordinary differential equation (ODE) models representing cellular dynamics and ion current across the membrane are integrated in the LIMPET library. openCARP is shipped with a set of commonly used models (including Courtemanche et al. [98] Koivumäki et al. [99], ten Tusscher et al. [100], O'Hara et al. [101]). The commandline tool bench is an interface for carrying out single cell experiments to tune models of cellular dynamics to given (patho-) physiological conditions. The most basic use case is to compute APs for a given membrane model. Advanced features of bench are to control stimulation, compute restitution curves under various protocols, to carry out voltage clamp experiments or to clamp arbitrary other state variables.

bench - -imp=Courtemanche --clamp-SVs=Ca_i --SV-clamp-files=Cai . dat

for example would clamp the intracellular calcium concentration of the Courtemanche et al. model to the time course defined in the Cai. dat file. The output files can either be processed with custom scripts or, ideally, preand postprocessing is integrated into a carputils Python script encoding the entire experiment including the call to bench. Besides CEP models, bench also provides myofilament models of active tension development. Often, one wants to change parameters of the cellular model to investigate how changes to a model component, due to e.g. drug effects, genetic mutations or disease-induced remodeling, affects behaviour. Parameters can easily be adjusted in LIMPET by specifying a value directly, or by using mathematical operations to alter the value relative to the default.

bench - -imp=COURTEMANCHE

--imp-par="Gto-65\%,GK1*2, GKs*2, GKur-50\%,GCaL-55\%,maxI_NaCa+60\%,maxCa_up*0.5,C_m+20\%"

for example would adjust the Courtemanche et al. model to reflect conditions of persistent atrial fibrillation-induced remodeling of cellular CEP [102] (Figure 4). The models equations are encoded in EasyML, a markup language developed for human readability. Changes to the model structure or parameters that are not exposed by default 


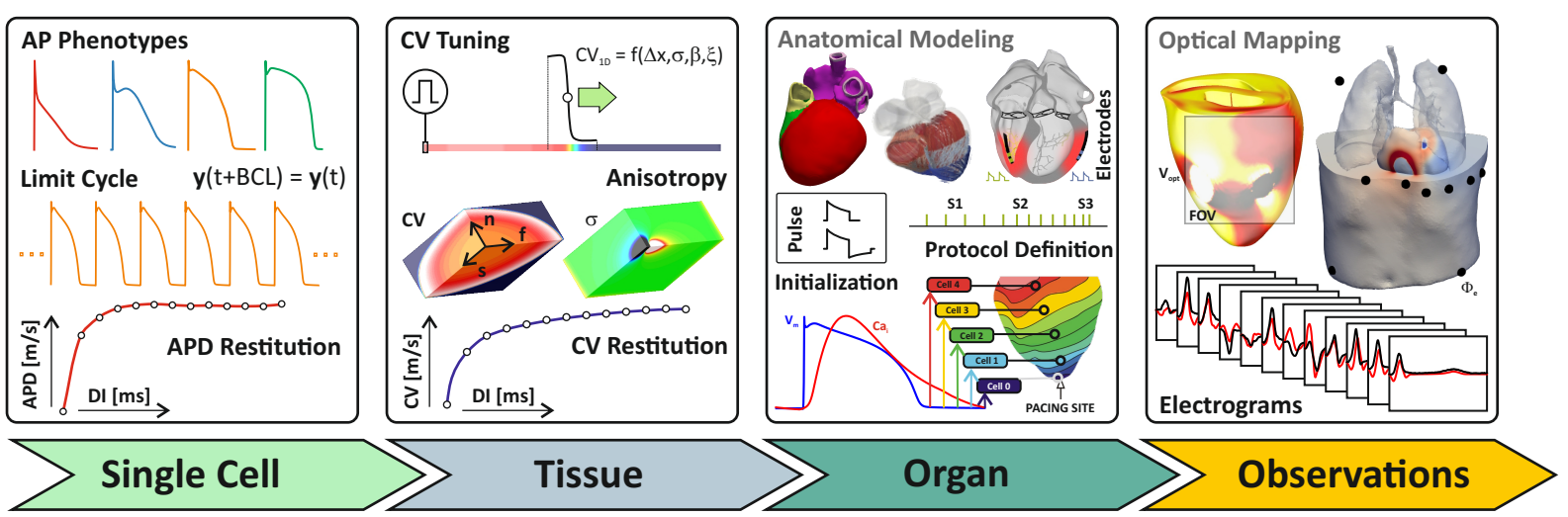

Figure 3: Overview of typical steps in an advanced CEP simulation study. Single Cell: AP phenotypes of different types of tissues are defined, paced to a stable limit cycle for a given set of cycle lengths, and dynamic properties such as APD restitution are evaluated. Initial state vectors $\boldsymbol{y}_{0}$ are stored for each phenotype. Tissue: Conductivities $\sigma_{\zeta}$ are determined for the mean spatial resolution $\Delta x$ of the organ-scale mesh, given surface-to-volume ratio, $\beta$, and numerical settings, $\xi$, by simulating uniform wavefront propagation in 1D along an eigenaxis, $\zeta$, to obtain the desired CVs along fiber, $\boldsymbol{f}$, sheet, $\boldsymbol{s}$, and sheet-normal, $\boldsymbol{n}$, direction, and anisotropy ratios between intra- and extracellular domain. CV restitution is evaluated then by measuring CV under increasingly shorter diastolic intervals. Organ: Organ models are generated using anatomical modeling pipelines, currently not included in openCARP, consisting of mesh generation, definition of fiber architecture, labeling of regions, computation of anatomical reference frames [95-97] and possibly the incorporation of a cardiac conduction system. Location and geometry of electrodes is defined, as are the pulse shapes used for stimulation and the pacing protocol. The organ model is then populated by assigning the ionic model initial states $\boldsymbol{y}_{0}$, as computed for each phenotype, to the respective tissues, and conductivities are assigned to the various regions to control CV. A pre-pacing protocol is applied to ascertain that the organ-scale model is as close as possible to a limit cycle for the given cycle length. Observations: Additional model components are set up to simulate observable data such as optical transmembrane voltages, $V_{\mathrm{opt}}$, invasively recorded electrograms $\Phi_{\mathrm{e}}$, and non-invasive ECGs recorded from the body surface.

can easily be implemented in the .model files directly. An automated way to produce shared libraries that can be loaded at run time allows using these custom-built models or adapt numerical time integration without having to recompile the entire simulator. Additional models can be included using CellML, the XML-based community standard for ionic models. Models downloaded from the CellML repository [51] can be converted to EasyML using tailored commandline scripts or interactively via the third-party tool Myokit [103].

Initial conditions for tissue scale simulations are generated with bench by creating a set of myocyte models representing the CEP heterogeneity at the tissue scale. Each myocyte model is paced at one or more basic cycle lengths based on the activation rates desired for the tissue model until the model settles in to a limit cycle. Initial state vectors of each myocyte model are stored, to be used later to populate the tissue and organ scale model as detailed in the limit cycle initialization example. Tailored experiments for interrogating and tuning of dynamic properties of ionic models such as APD restitution are also provided.

\subsubsection{Simple Tissue Level}

Simple geometries allow investigating fundamental mechanisms and determining properties of tissue CEP. These simple geometries play an important role in the generation and interpretation of modeling results as one can investigate basic mechanisms by isolating the effects of confounding factors, such as the prevailing myocyte orientation ("fiber orientation") or geometric complexities. Additionally, tissue behavioural properties can be quantified easily from these simplified models.

Mesh resolution has to strike a balance between computational effort and numerical accuracy. We refer to the $\mathrm{N}$-version monodomain benchmark for spatial convergence considerations [9]. Tissue and cellular level properties need tuning to replicate activation and repolarization sequences as observed in wet lab experiments or the clinic. 
bioRxiv preprint doi: https://doi.org/10.1101/2021.03.01.433036; this version posted June 10, 2021. The copyright holder for this preprint (which was not certified by peer review) is the author/funder, who has granted bioRxiv a license to display the preprint in perpetuity. It is made available under aCC-BY 4.0 International license.

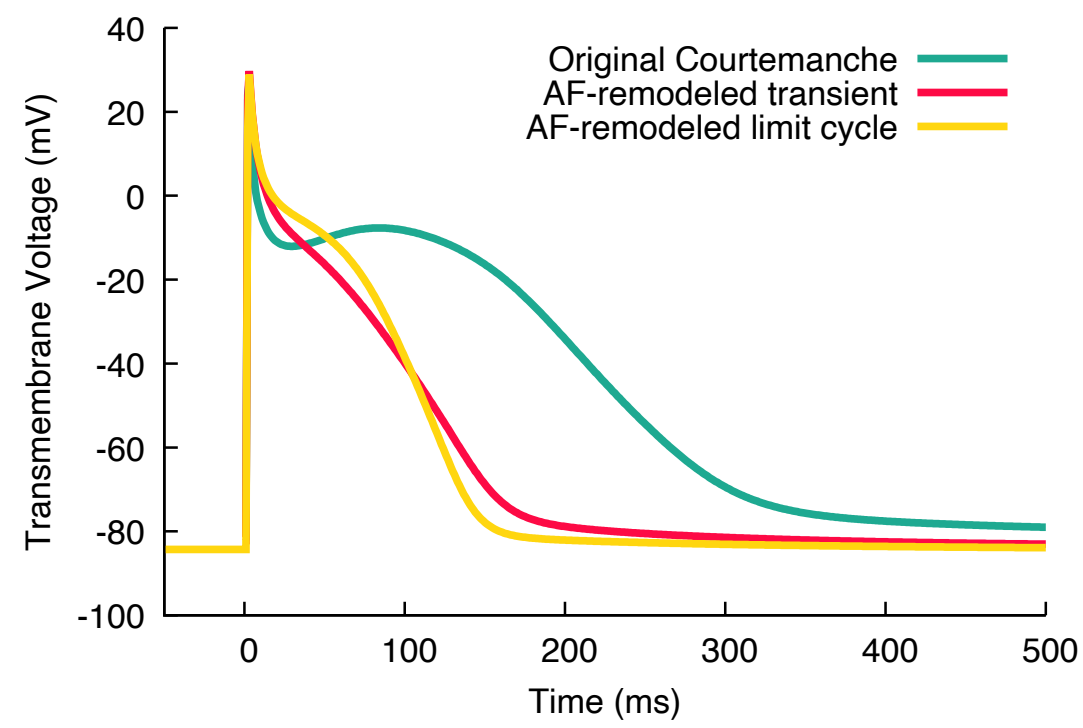

Figure 4: Action potentials of the original Courtemanche et al. [98] model (green) and remodelled variants reflecting persistent atrial fibrillation (AF) conditions [102]. Red: first action potential with the adapted parameters and initial state of the original model. Yellow: limit cycle AP after transient changes of the diastolic state equilibrated (100 stimuli at a basic cycle length of $1 \mathrm{~s}$ ).

Stimulation patterns initiate propagation with a velocity which varies as a function of space. The anisotropic $\mathrm{CV}$ is determined by myocyte orientation, the tissue conductivities along and across the myocytes, the surface-to-volume ratio, and the fast sodium current of the cells, but modified by the curvature of the activation wavefront, and the refractory state of the sodium channels. Technical factors related to spatial discretization and time integration also play a role, particularly when using coarser spatial resolutions [9]. Specifically, the tissue conductivities need to be determined to ensure physiological direction-dependent CVs before running more complex simulations. This is easily performed using the carputils tool tuneCV [104]. A wave front propagation in a 1D strand is simulated using the desired AP phenotype, and the $\mathrm{CV}$ measured at the center of the strand for given numerical settings and spatial resolution. Thus, tune $\mathrm{CV}$ compensates for artificial alterations in $\mathrm{CV}$ due to grid spacing, which is chosen to balance numerical accuracy and computational cost [9]. A comprehensive introduction to tune $C V$ can be found in the carputils example "Tuning Conduction Velocity". Other tissue properties like effective refractory period (ERP), vulnerable window or alternans can also be easily investigated in simple 1D geometries, for example, to extract arrhythmia predictors [28]. Additionally, rate dependent changes (restitution) of parameters provide insight into tissue behavior at fibrillatory activation rates. CV restitution is for example investigated in the carputils example "CV Restitution".

Simple 2D geometries can be used, for example, to investigate the effects of spatially heterogeneous tissue properties on arrhythmia dynamics. Spatial AP heterogeneity plays an import role in CEP as key features such as excitability, AP morphology and duration vary throughout the heart. Numerous effects of clinical relevance cannot be explained under the assumption of homogeneous tissue properties. Important examples are naturally existing heterogeneities within the ventricle that are responsible for the concordant T-wave in the ECG [105] and spatial changes of tissue conductivity (e.g. due to fibrosis) or/and electrophysiological properties in atrial fibrillation. In CEP modeling, two different approaches exist to account for spatial heterogeneity: either distinct regions are identified in which properties are uniform, or functions of space are defined which describe parameter variation, with granularity only being limited by spatial resolution. Region-based definition is, in general, easier to manage, but generates abrupt changes across region boundaries which may lead behavioural artifacts. Function-based assignment is more flexible and facilitates smooth variations that avoids potentially artificial high gradients, but is more challenging to define, particularly for complex anatomical models that, typically, require auxiliary anatomical coordinate systems to impose a desired variation [95-97]. The carputils example "EP Heterogeneity" illustrated the use of region-based heterogeneities, with four regions in which parameters can be set differently. Fig. 5A shows the impact of region-based variation in excitability upon the activation pattern. A comparison between region and gradient based heterogeneity is shown in another carputils example.

Key advantages of in silico CEP models over in vivo, ex vivo, or in vitro models are their ability to precisely control all parameters, to observe all quantities of interest at high spatiotemporal resolution, to test large parameter 
A

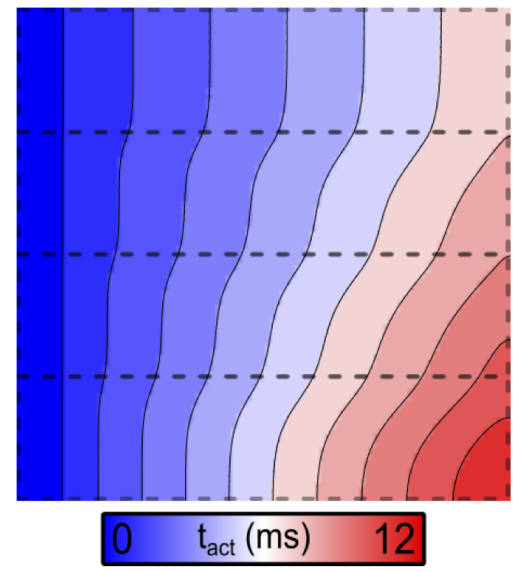

B

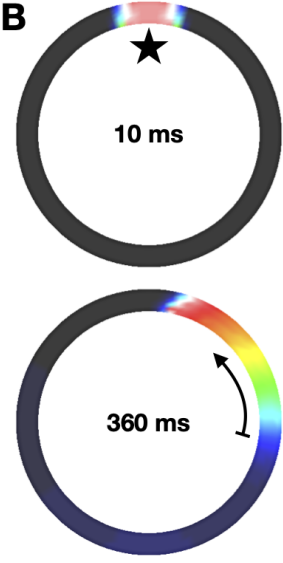

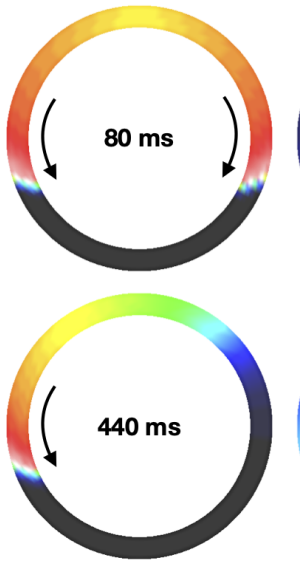

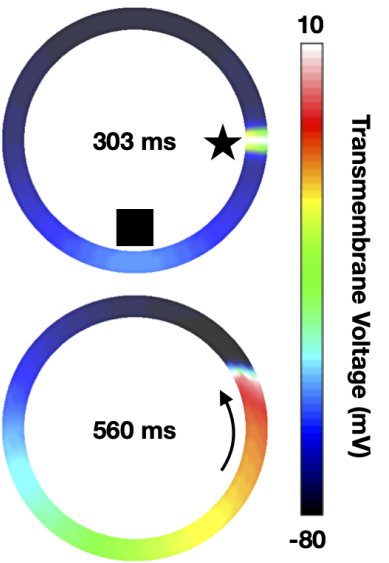

Figure 5: A: Activation times $\left(t_{a c t}\right)$ in a model with four regions of varying excitability. From the bottom to the top region, the conductance of the fast sodium channel $\left(g_{N a}\right)$ is set to $1 \mathrm{x}, 2 \mathrm{x}, 4 \mathrm{x}$, and $8 \mathrm{x} g_{N a}$ of the nominal value, respectively. Planar wave fronts initiated along the left edge of the sheet gradually distort due to these differences in CV. B: Initiation of a rotating wave in a ring model in a stylized representation of an atrial slice. The initial stimulus (delivered at $10 \mathrm{~ms}$, marked by a star) induces bidirectional propagation $(80 \mathrm{~ms})$. At the end of the refractory period $(303 \mathrm{~ms})$, a second stimulus delivered at a different site located at a critical recovery isoline initiates a wave front that is blocked from propagating downwards where tissue is still refractory (rectangle). This leads to unidirectional propagation upwards ( $360 \mathrm{~ms}$ ), setting up a sustained anatomical reentrant activation pattern $(440 \mathrm{~ms}$ and $560 \mathrm{~ms})$. This simulation was performed with ionic model settings corresponding to the persistent atrial fibrillation variant of the Courtemanche et al. model referred to above. In a healthy model, reentry cannot persist as the wavelength is too large for the given ring dimension.

spaces in a reproducible fashion, without being restricted by ethical concerns. Thus, mechanisms underlying CEP phenomena such as the formation and maintenance of an arrhythmia can be dissected in detail under a wide range of experimental settings. While organ-scale models are most comprehensive as they represent all factors at play, simplified 2D models are also highly relevant as these can provide crucial mechanistic insights with fewer confounding factors. Owing to their lighter weight, large parameter spaces can be probed and the observation of key variables and phenomena is more easily achieved than with the larger organ-scale models. Ring models representing a slice across a single cardiac chamber are a simple and popular 2D geometry for studying macroreentrant arrhythmias. Fig. 5B illustrates the induction process of a reentrant wave in such a model. Reentrant activation is only sustained if the wavelength, defined as $C V \times E R P$, is shorter than the perimeter of the ring. 2D sheet models can also be used to trace functional reentries, but their induction is less simple compared to the ring. The carputils example "Induction Protocols" presents four different methods to induce reentry in a sheet.

\subsubsection{Organ Level}

For whole chamber or organ level models, meshes must be created that accurately reflect the anatomy of the entity to be modelled. Streamlined workflows for this task are currently under development by various labs [106], typically relying on connecting heterogeneous software components, including commercial software, that are specialized for particular processing stages, ranging from multi-label segmentation, meshing, fiber architecture generation [107], anatomical reference frame generation [95-97] or topology generation of the specialized cardiac conduction system [108]. The Python-based carputils is perfectly suitable for creating such workflows [109]. However, owing to the technological heterogeneity of all this software, a complete integrated workflow is currently not available within carputils, but many basic elements required for building such workflows are. High quality anatomical models equipped with realistic fiber architecture, multiple label fields and pre-computed anatomical reference frames are becoming publicly available. A point in case is the recent study by Strocchi et al. [35] that placed in a repository a cohort of 24 volumetric four-chamber meshes derived from heart failure patients, which can be used for organ level simulations with openCARP. For cohort studies in healthy individuals, statistical shape models allow covering even more anatomical variability. Biventricular and biatrial shape models, together with 100 volumetric instances 
A

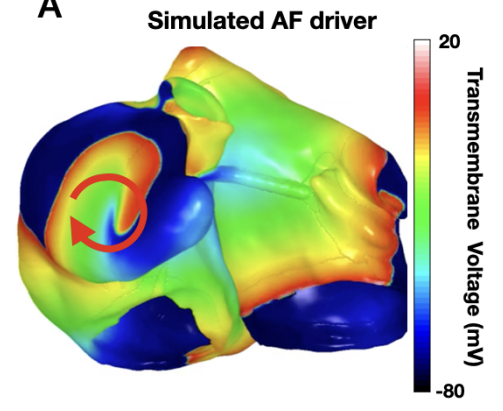

B

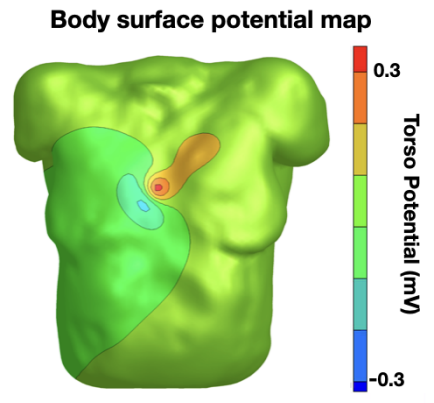

C

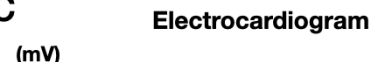

(mV)

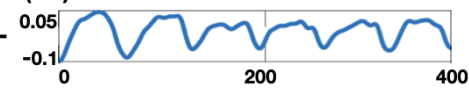

$\sum_{-0.06}^{0.04}$

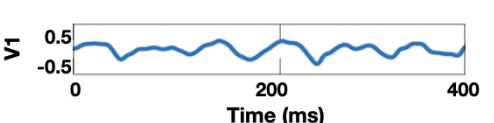

Figure 6: A: Biatrial volumetric model with ongoing reentry induced by pacing at the end of the refractory period using the carputils function model.induceReentry.PEERP(). B: Forward-calculated body surface potentials stemming from the transmembrane distribution in panel A. C: ECG (Einthoven lead I, Goldberger lead aVR and Wilson lead V1) extracted from the body surface potential map time series (panel B) reflecting the atrial fibrillation perpetuating at the tissue level (panel A).

ready to be used for openCARP simulation, are publicly available [110,111]. It is anticipated and foreseeable that the number of publicly available anatomical twin models will further increase, hopefully in part due to the standardization of formats under openCARP, yielding an abundant pool of cardiac anatomy models available to in silico CEP research. For creating digital twin models based on data acquired from individual patients model parameters need to be identified that minimize the misfit between simulated and observed quantities [112]. Owing to their mechanistic nature, such digital twin models show promise as an approach towards realizing precision medicine where therapeutic responses simulated in silico may be used to guide therapy planning and delivery. For this purpose, largely automated pipelines have been proposed for the atria $[72,113]$, the ventricles and the whole heart [35,53]. The geometric models can be augmented with population-level a priori knowledge regarding myocyte orientation in the atria [114,115], the ventricles [107] or the whole heart [116]. Like anatomy, functional properties can be represented by population averages [117] or personalized using non-invasive or intracardiac measurements $[72,79,118-121]$. Depending on the scientific question, relevant parameters can be global or spatial distributions, and can include, for example, tissue conductivity, ionic model properties, or the initially activated sites. Generic frames of reference, such as the universal ventricular coordinates [95] or universal atrial coordinates [122] allow parametrizing locations within the cardiac chambers to facilitate optimization approaches and transferability between models.

A common use case for organ level simulations is assessing the effects on arrhythmia inducibility of factors like fibrosis, scar or drugs. carputils provides several arrhythmia induction methods [123] to efficiently build such in silico experiments. The basic use is illustrated in one of the currently 28 carputils examples in addition to the API documentation. Users can readily integrate these induction methods in their own carputils experiments.

model.induceReentry.PEERP (..., stim_points, ...)

calls the carputils function PEERP to apply a protocol which paces at the end of ERP (PEERP) from all points included in the stim_points list. Depending on the cellular and tissue properties, reentry can be induced as shown in Fig. 6A where a rotor at the left atrial appendage drives reentry in both atria. Meshalyzer, part of the openCARP ecosystem, was used to produce Fig. 6A. Alternatively, openCARP results can be directly output in VTK format, or converted to VTK format in a post-processing step, for seamless visualization in ParaView [94].

\subsubsection{Body Level}

Cardiac excitation and the resulting spatially heterogeneous transmembrane voltage distribution, $V_{\mathrm{m}}(\boldsymbol{x}, t)$, in cardiac tissue act as sources that generate electric current flow in the extracellular domain comprising the interstitial space as well as a volume conductor potentially surrounding the cardiac tissue (blood pool, torso), often referred to as bath. Currents in the volume conductor set up an extracellular potential field, $\Phi_{\mathrm{e}}(\boldsymbol{x}, t)$, that can be sampled by electrodes to acquire body surface potentials or intracardiac electrograms. Apart from experimental settings where cardiac sources in the form of $V_{\mathrm{m}}(\boldsymbol{x}, t)$ can be observed more directly [124,125], extracellular potential recordings are the only means in the clinic to infer the cardiac source distribution. Owing to the unique importance of these 
signals for diagnosis and guiding therapy, methodologies facilitating their accurate simulation are an indispensable adjunct in many CEP simulation studies. These demands are reflected in the underlying bidomain equations solved by openCARP, which comprise both computational domains, the intracellular domain and the extracellular domain, thus facilitating the simulation of electrograms $[75,76,126]$ or torso potentials $[78,120]$. The electrical potential within the volume of interest can be computed in openCARP using different methods depending on the trade-off between computation and accuracy desired: i) The bidomain setting, which considers bath-loading effects [127] and allows for bidirectional interplay between intracellular and extracellular potential fields; ii) Alternatively, extracellular potential can be recovered in a post-processing step from a given spatiotemporal transmembrane voltage distribution obtained through, a monodomain simulation. Bishop \& Plank [128] showed that the latter approach (referred to as pseudo bidomain) retains accuracy in most cases, particularly with augmentation techniques to recover bath-loading effects close to the tissue-bath interface [129], while drastically reducing the computational cost; and iii) An even more simplistic approach is to assume that the cardiac tissue is immersed in a homogeneous volume conductor of infinite size, which allows recovering the extracellular potential $\Phi_{\mathrm{e}}$ at specific points in space from a given transmembrane voltage distribution by using the integral solution of Poisson's equation. One of the carputils examples introduces all three methods and involves the user in simple experiments highlighting the differences between them. To compute surface potentials in own carputils experiments, the ep module provides the model_type_opts (sourceModel) function to choose between $\Phi_{\mathrm{e}}$ recovery, pseudo-bidomain and bidomain. Fig. 6B shows a snapshot of the body surface potential map generated by the transmembrane voltage distribution $V_{\mathrm{m}}$ during atrial fibrillation shown in Fig. 6A. By extracting the time series of extracellular potentials $\Phi_{\mathrm{e}}(t)$ at the electrode locations, virtual ECGs can be derived by subtraction of electrograms along the lead axes (Fig. 6C).

\section{Discussion}

openCARP is a CEP simulation environment for carrying out advanced in silico experiments. The openCARP framework is highly versatile and comprehensive, in principle allowing replicating and building on the vast majority of published CEP simulation studies which rely only on the monodomain, bidomain and Poisson equations. openCARP builds on the core technologies of its proprietary predecessors, CARP and acCELLerate, that have matured over 20+ years of cutting edge modeling research, having been used in more than 250 published studies. openCARP provides a convenient and flexible user interface that enables performing complex simulations, requiring little to no programming experience. It supports the full research life cycle from exploration through to conclusive analysis and publication, to archiving and sharing of data, experimental protocols, models and source code. As such, openCARP can be a suitable software solution for a large portion of the CEP community, by contributing to the use, transparency, standardization and reproducibility of in silico approaches.

openCARP is designed to appeal to users of all experience levels. New users can easily explore parameter effects in prebuilt carputils experiments, while more experienced users can further extend such experiments to more elaborate scenarios, or build their own complex experiments from scratch. openCARP provides a solution for potential new users in the fields of basic science CEP (integrating experimental data into mechanistic models), cardiology research (understanding arrhythmia mechanisms, diagnostic support, therapy stratification, planning and delivery), pharmaceutical companies (virtual testing arrhythmogenic potency of new compounds), medical device companies (interpreting measured signals, optimizing new device designs [130], testing their safety and efficacy, and understanding reasons for device failure [26]), educational use (visualizing complex heart function), and regulatory entities (e.g., the FDA which is already encouraging the use of numerical models for drug and device testing [2]).

The central resource and entry point is the project web page www . openCARP. org. From there, the community has access to the user and developer documentation, tutorials, examples, the GitLab projects, and a question and answer system [131]. Besides the online platform, in-person contact among and between users and developers is recognized as a key factor to build and maintain a strong community. Regular user meetings allow training new users, exchange experiences with the software, and stay close with the community as part of a continuous feedback loop. Within a year from the release of the first public version, more than 250 users opened an account in our user and development community.

openCARP is a meritocratic, consensus-based community project. Anyone with an interest in the project can join the community, contribute to the project design, and participate in the decision making process. Community contributions are highly appreciated and can range from code commits to bug reports, suggestion of ideas and new directions for improving the software, documentation or any aspect of the community platform. Code contributions will enter a review process as a quality control measure and will be integrated in the code base if they meet quality criteria and satisfy a community need. The roles in the openCARP project are users, developers, maintainers and the steering committee. Users are community members who have a need for the project. The openCARP project asks its users to participate in the project and community as much as possible. Contributors engage with the project 
in concrete ways, through the issue tracker, question and answer system, or by writing or editing documentation. They submit changes to the project itself via commits to non-protected branches of our git repositories, which then undergo review by the maintainers before be merged into the main branch of code. Most of the 12 maintainers and five steering committee members are employed on tenured stable academic positions, ensuring long-term maintenance and support.

An important prerequisite for the reproducibility and reusability of models and associated simulation data is their enrichment with metadata, and publication as FAIR [132] and open data. While openCARP carries the features to reproduce the majority of published CEP simulations, the practical limitation is a not fully complete description of experiments or limited data availability. We are, therefore, currently embedding mechanisms in carputils to automate the extraction of all relevant information for a specific experiment, and create packages suitable for archiving and sharing. A convenient interface to upload these simulation packages to an openCARP specific section of the RADAR4KIT research data repository [133] will be provided in the near future. RADAR4KIT is a long-term research data repository that adheres to the Open Archival Information System (OAIS) standard [134]. It provides access control (public, private sharing via link), persistent DOI identifiers, standardized interfaces facilitating data harvesting, and is integrated with research data meta repositories like re3data [135]. In this way, the carputils experiment can be further evaluated by the scientific community, and future research can build upon it. Errors or previously undiscovered artifacts can be found and described, leading to improved data and research quality in the long term as one of the main goals of open science.

In conclusion, openCARP is an open CEP simulator released to the academic community to advance the computational CEP field by making state-of-the-art simulations accessible. A commercial license can be requested. In combination with the open source carputils framework and additional open source software around it, it offers a tailored software solution for the scientific community in the CEP field and contributes towards increasing use, transparency, standardization and reproducibility of in silico experiments.

\section{Acknowledgments}

We gratefully acknowledge support by Deutsche Forschungsgemeinschaft (DFG) (project ID 391128822, LO 2093/1-1, GS 1758/4-1). We further acknowledge the software contributions of NumeriCor GmbH, Graz, Austria who conceived and implemented the openCARP simulation core. We thank Felix Bach, Jochen Klar and Robert Ulrich for developing and providing the openCARP development environment as well as Michael Selzer and Philipp Zschumme for developing and providing toolcompendium for generation of user documentation. We thank Prof. Natalia Trayanova for agreeing to make the EasyML model generator, developed in her lab by Rob Blake, available to be distributed with openCARP. We thank Giorgio Luongo for providing figures of simulation results.

\section{Conflicts of interest}

GP, AN, CA and EJV are co-founders of NumeriCor GmbH. All other authors declare no conflict of interest.

\section{References}

[1] S. A. Niederer, J. Lumens, and N. A. Trayanova, "Computational models in cardiology." Nature Reviews. Cardiology, 10 2018. doi:10.1038/s41569-018-0104-y

[2] US Food and Drug Administration, "Reporting of computational modeling studies in medical device submissions," 2016.

[3] J. Corral-Acero, F. Margara, M. Marciniak et al., "The 'digital twin' to enable the vision of precision cardiology." European heart journal, 3 2020. doi:10.1093/eurheartj/ehaa159

[4] D. G. Whittaker, R. A. Capel, M. Hendrix, X. H. S. Chan, N. Herring, N. J. White, G. R. Mirams, and R.-A. B. Burton, "Cardiac TdP risk stratification modelling of anti-infective compounds including chloroquine and hydroxychloroquine," Royal Society Open Science, vol. 8, no. 4, Apr. 2021. doi:10.1098/rsos.210235. https://doi.org/10.1098/rsos.210235

[5] S. Gaeta, T. D. Bahnson, and C. Henriquez, "Mechanism and magnitude of bipolar electrogram directional sensitivity: Characterizing underlying determinants of bipolar amplitude," Heart Rhythm, vol. 17, no. 5, pp. 777-785, May 2020. doi:10.1016/j.hrthm.2019.12.010. https://doi.org/10.1016/j.hrthm.2019.12.010 
[6] F. Margara, Z. J. Wang, F. Levrero-Florencio, A. Santiago, M. Vázquez, A. Bueno-Orovio, and B. Rodriguez, "In-silico human electro-mechanical ventricular modelling and simulation for drug-induced pro-arrhythmia and inotropic risk assessment," Progress in Biophysics and Molecular Biology, vol. 159, pp. 58-74, Jan. 2021. doi:10.1016/j.pbiomolbio.2020.06.007. https://doi.org/10.1016/j.pbiomolbio.2020.06.007

[7] M. Paci, J. T. Koivumäki, H. R. Lu, D. J. Gallacher, E. Passini, and B. Rodriguez, "Comparison of the simulated response of three in silico human stem cell-derived cardiomyocytes models and in vitro data under 15 drug actions," Frontiers in Pharmacology, vol. 12, Mar. 2021. doi:10.3389/fphar.2021.604713. https://doi.org/10.3389/fphar.2021.604713

[8] A. Gharaviri, S. Pezzuto, M. Potse, S. Verheule, G. Conte, R. Krause, U. Schotten, and A. Auricchio, "Left atrial appendage electrical isolation reduces atrial fibrillation recurrences," Circulation: Arrhythmia and Electrophysiology, vol. 14, no. 1, Jan. 2021. doi:10.1161/circep.120.009230. https://doi.org/10.1161/circep.120.009230

[9] S. A. Niederer, E. Kerfoot, A. P. Benson et al., "Verification of cardiac tissue electrophysiology simulators using an n-version benchmark," Philosophical Transactions of the Royal Society A: Mathematical, Physical and Engineering Sciences, vol. 369, no. 1954, pp. 4331-4351, 2011. doi:10.1098/rsta.2011.0139

[10] R. C. Barr and R. Plonsey, "Propagation of excitation in idealized anisotropic two-dimensional tissue." Biophys. J., vol. 45, no. 6, pp. 1191-202, jun 1984. doi:10.1016/S0006-3495(84)84268-X

[11] M. S. Spach and J. M. Kootsey, "Relating the sodium current and conductance to the shape of transmembrane and extracellular potentials by simulation: effects of propagation boundaries." IEEE Trans. Biomed. Eng., vol. 32, no. 10, pp. 743-55, oct 1985. doi:10.1109/TBME.1985.325489

[12] B. Baillargeon, N. Rebelo, D. D. Fox, R. L. Taylor, and E. Kuhl, "The Living Heart Project: A robust and integrative simulator for human heart function," Eur. J. Mech. - A/Solids, pp. 1-10, apr 2014. doi:10.1016/j.euromechsol.2014.04.001

[13] M. J. Bishop and G. Plank, "The role of fine-scale anatomical structure in the dynamics of reentry in computational models of the rabbit ventricles." J Physiol, vol. 590, no. Pt 18, pp. 4515-4535, sep 2012. doi:10.1113/jphysiol.2012.229062

[14] M. Strocchi, A. W. Lee, A. Neic et al., "His Bundle and Left Bundle Pacing with Optimised Atrio-ventricular Delay Achieve Superior Electrical Synchrony over Endocardial and Epicardial Pacing in Left Bundle Branch Block Patients," Hear. Rhythm, 2020. doi:10.1016/j.hrthm.2020.06.028

[15] E. J. Vigmond and L. J. Leon, "Computationally efficient model for simulating electrical activity in cardiac tissue with fiber rotation." Ann. Biomed. Eng., vol. 27, no. 2, pp. 160-70, 1999. doi:10.1114/1.160

[16] E. J. Vigmond, M. Hughes, G. Plank, and L. J. Leon, "Computational tools for modeling electrical activity in cardiac tissue," J Electrocardiol, vol. 36 Suppl, pp. 69-74, 2003. doi:10.1016/j.jelectrocard.2003.09.017

[17] G. Seemann, F. B. Sachse, M. Karl, D. L. Weiss, V. Heuveline, and O. Dössel, "Framework for modular, flexible and efficient solving the cardiac bidomain equation using PETSc," Mathematics in Industry, vol. 15, no. 2, pp. 363-369, 1 2010. doi:10.1007/978-3-642-12110-4_55

[18] C. Bradley, A. Bowery, R. Britten et al., "OpenCMISS: a multi-physics \& multi-scale computational infrastructure for the VPH/physiome project." Progress in biophysics and molecular biology, vol. 107, no. 1, pp. 32-47, 10 2011. doi:10.1016/j.pbiomolbio.2011.06.015

[19] M.-C. Trudel, B. Dubé, M. Potse, R. M. Gulrajani, and L. J. Leon, "Simulation of QRST integral maps with a membrane-based computer heart model employing parallel processing." IEEE transactions on bio-medical engineering, vol. 51, no. 8, pp. 1319-29, 8 2004. doi:10.1109/TBME.2004.827934

[20] M. B. Liu, E. de Lange, A. Garfinkel, J. N. Weiss, and Z. Qu, "Delayed afterdepolarizations generate both triggers and a vulnerable substrate promoting reentry in cardiac tissue." Heart rhythm, vol. 12, no. 10, pp. 2115-24, 10 2015. doi:10.1016/j.hrthm.2015.06.019

[21] E. A. Heidenreich, J. M. Ferrero, M. Doblaré, and J. F. Rodríguez, "Adaptive macro finite elements for the numerical solution of monodomain equations in cardiac electrophysiology," Ann. Biomed. Eng., vol. 38, no. 7, pp. 2331-2345, 2010. doi:10.1007/s10439-010-9997-2

[22] K. P. Vincent, M. J. Gonzales, A. K. Gillette, C. T. Villongco, S. Pezzuto, J. H. Omens, M. J. Holst, and A. D. McCulloch, "High-order finite element methods for cardiac monodomain simulations." Front. Physiol., vol. 6, no. August, p. 217, 2015. doi:10.3389/fphys.2015.00217 
[23] F. H. Fenton, E. M. Cherry, H. M. Hastings, and S. J. Evans, "Multiple mechanisms of spiral wave breakup in a model of cardiac electrical activity," Chaos (Woodbury, N.Y.), vol. 12, no. 3, pp. 852-892, 12002. doi: $10.1063 / 1.1504242$

[24] M. J. Bishop, E. J. Vigmond, and G. Plank, "The functional role of electrophysiological heterogeneity in the rabbit ventricle during rapid pacing and arrhythmias." Am. J. Physiol. Heart Circ. Physiol., vol. 304, no. 9, pp. H1240-52, 2013. doi:10.1152/ajpheart.00894.2012

[25] A. Loewe, E. Poremba, T. Oesterlein, A. Luik, C. Schmitt, G. Seemann, and O. Dössel, "Patient-specific identification of atrial flutter vulnerability-a computational approach to reveal latent reentry pathways," Frontiers in Physiology, vol. 9, no. Article 1910, 1 2019. doi:10.3389/fphys.2018.01910

[26] D. J. Swenson, R. T. Taepke, J. J. Blauer, E. Kwan, E. Ghafoori, G. Plank, E. Vigmond, R. S. MacLeod, P. DeGroot, and R. Ranjan, "Direct comparison of a novel antitachycardia pacing algorithm against present methods using virtual patient modeling," Hear. Rhythm, vol. 17, no. 9, pp. 1602-1608, 2020. doi:10.1016/j.hrthm.2020.05.009

[27] G. Plank, L. J. Leon, S. Kimber, and E. J. Vigmond, "Defibrillation depends on conductivity fluctuations and the degree of disorganization in reentry patterns." J. Cardiovasc. Electrophysiol., vol. 16, no. 2, pp. 205-16, feb 2005. doi:10.1046/j.1540-8167.2005.40140.x

[28] A. Loewe, Y. Lutz, M. Wilhelms, D. Sinnecker, P. Barthel, E. P. Scholz, O. Dössel, G. Schmidt, and G. Seemann, "In-silico assessment of the dynamic effects of amiodarone and dronedarone on human atrial patho-electrophysiology." Europace, vol. 16, no. S4, pp. iv30-iv38, 11 2014. doi:10.1093/europace/euu230

[29] H. Anzt, F. Bach, S. Druskat et al., "An environment for sustainable research software in germany and beyond: current state, open challenges, and call for action," F1000Research, vol. 9, p. 295, 12020. doi:10.12688/f1000research.23224.1

[30] G. W. Beeler and H. Reuter, "Reconstruction of the action potential of ventricular myocardial fibers," Journal of Physiology, vol. 268, pp. 177-210, 1 1977. doi:10.1113/jphysiol.1977.sp011853

[31] L. Ebihara and E. A. Johnson, "Fast sodium current in cardiac muscle. a quantitative description," Biophysical Journal, vol. 32, pp. 779-790, 1 1980. doi:10.1016/S0006-3495(80)85016-8

[32] C. H. Luo and Y. Rudy, "A model of the ventricular cardiac action potential. depolarization, repolarization, and their interaction," Circulation Research, vol. 68, pp. 1501-1526, 1 1991. doi:10.1161/01.RES.68.6.1501

[33] E. J. Vigmond, F. Aguel, and N. a. Trayanova, "Computational techniques for solving the bidomain equations in three dimensions." IEEE Trans. Biomed. Eng., vol. 49, no. 11, pp. 1260-9, nov 2002. doi:10.1109/TBME.2002.804597

[34] D. M. Harrild and C. S. Henriquez, "A computer model of the normal conduction in the human atria," Circ. Res., 2004. doi:10.1161/01.RES.0000148636.60732.2e

[35] M. Strocchi, C. M. Augustin, M. A. F. Gsell et al., "A publicly available virtual cohort of four-chamber heart meshes for cardiac electro-mechanics simulations." PloS one, vol. 15, no. 6, p. e0235145, 12020. doi:10.1371/journal.pone. 0235145

[36] S. R. Kharche, E. Vigmond, I. R. Efimov, and H. Dobrzynski, "Computational assessment of the functional role of sinoatrial node exit pathways in the human heart." PloS one, vol. 12, no. 9, p. e0183727, 12017. doi:10.1371/journal.pone.0183727

[37] A. Loewe, Y. Lutz, N. Nagy, A. Fabbri, C. Schweda, A. Varro, and S. Severi, "Inter-species differences in the response of sinus node cellular pacemaking to changes of extracellular calcium," in 41st Annual International Conference of the IEEE Engineering in Medicine and Biology Society (EMBC), 2019, pp. 1875-1878. doi:10.1109/EMBC.2019.8857573

[38] A. Loewe, Y. Lutz, D. Nairn et al., "Hypocalcemia-induced slowing of human sinus node pacemaking," Biophysical Journal, vol. 117, no. 12, pp. 2244-2254, 1 2019. doi:10.1016/j.bpj.2019.07.037

[39] J. Li, I. D. Greener, S. Inada et al., "Computer three-dimensional reconstruction of the atrioventricular node." Circulation research, vol. 102, no. 8, pp. 975-85, 4 2008. doi:10.1161/CIRCRESAHA.108.172403

[40] E. J. Vigmond and B. D. Stuyvers, "Modeling our understanding of the his-purkinje system." Progress in biophysics and molecular biology, vol. 120, no. 1-3, pp. 179-88, 1 2016. doi:10.1016/j.pbiomolbio.2015.12.013 
[41] A. Loewe, E. M. Wülfers, and G. Seemann, "Cardiac ischemia-insights from computational models." Herzschrittmachertherapie \& Elektrophysiologie, vol. 29, no. 1, pp. 48-56, 3 2018. doi:10.1007/s00399017-0539-6

[42] C. M. Costa, A. Neic, E. Kerfoot et al., "Pacing in proximity to scar during cardiac resynchronization therapy increases local dispersion of repolarization and susceptibility to ventricular arrhythmogenesis." Heart rhythm, 3 2019. doi:10.1016/j.hrthm.2019.03.027

[43] C. H. Roney, J. D. Bayer, S. Zahid, M. Meo, P. M. J. Boyle, N. A. Trayanova, M. Haïssaguerre, R. Dubois, H. Cochet, and E. J. Vigmond, "Modelling methodology of atrial fibrosis affects rotor dynamics and electrograms." Europace, vol. 18, no. suppl 4, pp. iv146-iv155, 12 2016. doi:10.1093/europace/euw365

[44] A. Jadidi, M. Nothstein, J. Chen et al., "Specific electrogram characteristics identify the extra-pulmonary vein arrhythmogenic sources of persistent atrial fibrillation - characterization of the arrhythmogenic electrogram patterns during atrial fibrillation and sinus rhythm.” Scientific Reports, vol. 10, no. 1, p. 9147, 62020. doi:10.1038/s41598-020-65564-2

[45] G. Balaban, B. P. Halliday, C. Mendonca Costa, W. Bai, B. Porter, C. A. Rinaldi, G. Plank, D. Rueckert, S. K. Prasad, and M. J. Bishop, "Fibrosis microstructure modulates reentry in non-ischemic dilated cardiomyopathy: Insights from imaged guided 2d computational modeling," Frontiers in Physiology, vol. 9, 1 2018. doi:10.3389/fphys.2018.01832

[46] L. Zhou, S. Solhjoo, B. Millare, G. Plank, M. R. Abraham, S. Cortassa, N. Trayanova, and B. O'Rourke, "Effects of regional mitochondrial depolarization on electrical propagation: implications for arrhythmogenesis." Circ. Arrhythm. Electrophysiol., vol. 7, no. 1, pp. 143-51, 2014. doi:10.1161/CIRCEP.113.000600

[47] G. Plank, R. A. B. Burton, P. Hales et al., "Generation of histo-anatomically representative models of the individual heart: tools and application," Philos Trans A Math Phys Eng Sci, vol. 367, no. 1896, pp. 2257-2292, 2009. doi:10.1098/rsta.2009.0056

[48] M. J. Bishop, G. Plank, R. A. B. Burton, J. E. Schneider, D. J. Gavaghan, V. Grau, and P. Kohl, “Development of an anatomically detailed MRI-derived rabbit ventricular model and assessment of its impact on simulations of electrophysiological function." American Journal of Physiology. Heart and Circulatory Physiology, vol. 298, no. 2, pp. H699-718, 2 2010. doi:10.1152/ajpheart.00606.2009

[49] G. Seemann, A. Loewe, and E. M. Wülfers, "Effects of fibroblasts coupling on the electrophysiology of cardiomyocytes from different regions of the human atrium: A simulation study," in Computing in Cardiology, vol. 44, 2017. doi:10.22489/CinC.2017.380-451

[50] C. M. Costa, F. O. Campos, A. J. Prassl, R. W. dos Santos, D. Sanchez-Quintana, H. Ahammer, E. Hofer, and G. Plank, "An efficient finite element approach for modeling fibrotic clefts in the heart," IEEE Transactions on Biomedical Engineering, vol. 61, no. 3, pp. 900-910, 1 2014. doi:10.1109/TBME.2013.2292320

[51] T. Yu, C. M. Lloyd, D. P. Nickerson et al., "The Physiome Model Repository 2," Bioinformatics, vol. 27, no. 5, pp. 743-744, 01 2011. doi:10.1093/bioinformatics/btq723

[52] A. J. Prassl, F. Kickinger, H. Ahammer, V. Grau, J. E. Schneider, E. Hofer, E. J. Vigmond, N. a. Trayanova, and G. Plank, "Automatically generated, anatomically accurate meshes for cardiac electrophysiology problems," IEEE Trans. Biomed. Eng., vol. 56, no. 5, pp. 1318-1330, may 2009. doi:10.1109/TBME.2009.2014243

[53] A. Crozier, C. M. Augustin, A. Neic et al., "Image-based personalization of cardiac anatomy for coupled electromechanical modeling," Annals of Biomedical Engineering, 1 2015. doi:10.1007/s10439-015-1474-5

[54] A. Neic, M. A. Gsell, E. Karabelas, A. J. Prassl, and G. Plank, "Automating image-based mesh generation and manipulation tasks in cardiac modeling workflows using meshtool," SoftwareX, vol. 11, p. 100454, 1 2020. doi:10.1016/j.softx.2020.100454

[55] M. L. Hines and N. T. Carnevale, "NEURON: a tool for neuroscientists." The Neuroscientist : a review journal bringing neurobiology, neurology and psychiatry, vol. 7, no. 2, pp. 123-35, 42001. doi: $10.1177 / 107385840100700207$

[56] G. M. J. Barca, C. Bertoni, L. Carrington et al., "Recent developments in the general atomic and molecular electronic structure system," The Journal of Chemical Physics, vol. 152, no. 15, p. 154102, Apr. 2020. doi: $10.1063 / 5.0005188$ 
[57] G. R. Mirams, C. J. Arthurs, M. O. Bernabeu et al., "Chaste: an open source c++ library for computational physiology and biology," PLoS Computational Biology, vol. 9, no. 3, p. e1002970, 12013. doi:10.1371/journal.pcbi.1002970

[58] S. Pezzuto, F. W. Prinzen, M. Potse, F. Maffessanti, F. Regoli, M. L. Caputo, G. Conte, R. Krause, and A. Auricchio, "Reconstruction of three-dimensional biventricular activation based on the 12-lead electrocardiogram via patient-specific modelling," EP Europace, 1 2020. doi:10.1093/europace/euaa330

[59] A. Quarteroni, T. Lassila, S. Rossi, and R. Ruiz-Baier, "Integrated heart-coupling multiscale and multiphysics models for the simulation of the cardiac function," Computer Methods in Applied Mechanics and Engineering, vol. 314, pp. 345-407, 1 2017. doi:10.1016/j.cma.2016.05.031

[60] P. Colli Franzone, L. F. Pavarino, and S. Scacchi, "A numerical study of scalable cardiac electro-mechanical solvers on HPC architectures," Frontiers in Physiology, vol. 9, 1 2018. doi:10.3389/fphys.2018.00268

[61] R. H. Clayton and A. V. Holden, "Propagation of normal beats and re-entry in a computational model of ventricular cardiac tissue with regional differences in action potential shape and duration," Prog. Biophys. Mol. Biol., vol. 85, no. 2-3, pp. 473-499, 1 2004. doi:10.1016/j.pbiomolbio.2003.12.002

[62] J. Sundnes, S. Wall, H. Osnes, T. Thorvaldsen, and A. D. McCulloch, "Improved discretisation and linearisation of active tension in strongly coupled cardiac electro-mechanics simulations," Computer Methods in Biomechanics and Biomedical Engineering, vol. 17, no. 6, pp. 604-615, 12014. doi:10.1080/10255842.2012.704368

[63] M. Antonioletti, V. N. Biktashev, A. Jackson, S. R. Kharche, T. Stary, and I. V. Biktasheva, "Beatbox-hpc simulation environment for biophysically and anatomically realistic cardiac electrophysiology," PLOS ONE, vol. 12, no. 5, pp. 1-37, 05 2017. doi:10.1371/journal.pone.0172292

[64] J. Koehler Leman, B. D. Weitzner, P. D. Renfrew et al., "Better together: Elements of successful scientific software development in a distributed collaborative community," PLOS Computational Biology, vol. 16, no. 5, pp. 1-20, 05 2020. doi:10.1371/journal.pcbi.1007507. https://doi.org/10.1371/journal.pcbi.1007507

[65] B. Shneiderman, Designing the User Interface: Strategies for Effective Human-Computer Interaction, 3rd ed. USA: Addison-Wesley Longman Publishing Co., Inc., 1997. ISBN 0201694972

[66] G. Wang and B. Peng, "Script of scripts: A pragmatic workflow system for daily computational research," PLOS Computational Biology, vol. 15, no. 2, pp. 1-14, 02 2019. doi:10.1371/journal.pcbi.1006843. https://doi.org/10.1371/journal.pcbi.1006843

[67] D. S. Katz, L. C. McInnes, D. E. Bernholdt et al., "Community organizations: Changing the culture in which research software is developed and sustained," Computing in Science \& Engineering, vol. 21, pp. 8-24, 3 2019. doi:10.1109/MCSE.2018.2883051

[68] S. Niederer, L. Mitchell, N. Smith, and G. Plank, "Simulating human cardiac electrophysiology on clinical time-scales," Frontiers in Physiology, vol. 2, p. 14, 2011. doi:10.3389/fphys.2011.00014. https://www.frontiersin.org/article/10.3389/fphys.2011.00014

[69] B. D. Lee, “Ten simple rules for documenting scientific software," PLOS Computational Biology, vol. 14, no. 12, pp. 1-6, 12 2018. doi:10.1371/journal.pcbi.1006561. https://doi.org/10.1371/journal.pcbi.1006561

[70] W. J. Schroeder, K. Martin, and B. Lorensen, The visualization toolkit, 4th ed. Kitware, 2006.

[71] E. J. Vigmond, R. Weber dos Santos, A. J. Prassl, M. Deo, and G. Plank, "Solvers for the cardiac bidomain equations," Progress in Biophysics and Molecular Biology, vol. 96, no. 1-3, pp. 3-18, 12008. doi:10.1016/j.pbiomolbio.2007.07.012

[72] M. W. Krueger, G. Seemann, K. Rhode, D. U. J. Keller, C. Schilling, A. Arujuna, J. Gill, M. D. O’Neill, R. Razavi, and O. Dössel, "Personalization of atrial anatomy and electrophysiology as a basis for clinical modeling of radio-frequency ablation of atrial fibrillation," IEEE Transactions on Medical Imaging, vol. 32, no. 1, pp. 73-84, 1 2013. doi:10.1109/TMI.2012.2201948

[73] F. O. Campos, Y. Shiferaw, A. J. Prassl, P. M. Boyle, E. J. Vigmond, and G. Plank, "Stochastic spontaneous calcium release events trigger premature ventricular complexes by overcoming electrotonic load." Cardiovascular research, vol. 107, no. 1, pp. 175-83, 7 2015. doi:10.1093/cvr/cvv149

[74] A. Loewe, M. Wilhelms, F. Fischer, E. P. Scholz, O. Dössel, and G. Seemann, "Arrhythmic potency of human ether-a-go-go-related gene mutations $1532 \mathrm{p}$ and n588k in a computational model of human atrial myocytes," Europace, vol. 16, no. 3, pp. 435-443, 1 2014. doi:10.1093/europace/eut375 
[75] E. Vigmond, A. Pashaei, S. Amraoui, H. Cochet, and M. Hassaguerre, "Percolation as a mechanism to explain atrial fractionated electrograms and reentry in a fibrosis model based on imaging data," Heart Rhythm : the Official Journal of the Heart Rhythm Society, vol. 13, no. 7, pp. 1536-1543, 12016. doi:10.1016/j.hrthm.2016.03.019

[76] M. Alessandrini, M. Valinoti, L. Unger, T. Oesterlein, O. Dössel, C. Corsi, A. Loewe, and S. Severi, "A computational framework to benchmark basket catheter guided ablation in atrial fibrillation," Frontiers in Physiology, vol. 9, p. 1251, 1 2018. doi:10.3389/fphys.2018.01251

[77] H. J. Arevalo, F. Vadakkumpadan, E. Guallar, A. Jebb, P. Malamas, K. C. Wu, and N. A. Trayanova, "Arrhythmia risk stratification of patients after myocardial infarction using personalized heart models," Nature Communications, vol. 7, p. 11437, 1 2016. doi:10.1038/ncomms11437

[78] R. Andlauer, G. Seemann, L. Baron, O. Dössel, P. Kohl, P. Platonov, and A. Loewe, "Influence of left atrial size on p-wave morphology: differential effects of dilation and hypertrophy," Europace, vol. 20, no. S3, pp. iii36-iii44, 11 2018. doi:10.1093/europace/euy231

[79] A. Prakosa, H. J. Arevalo, D. Deng et al., "Personalized virtual-heart technology for guiding the ablation of infarct-related ventricular tachycardia," Nature Biomedical Engineering, 1 2018. doi:10.1038/s41551-018$0282-2$

[80] H. Lehrmann, A. S. Jadidi, J. Minners, J. Chen, B. Müller-Edenborn, R. Weber, O. Dössel, T. Arentz, and A. Loewe, "Novel electrocardiographic criteria for real-time assessment of anterior mitral line block," JACC: Clinical Electrophysiology, vol. 4, no. 7, pp. 920-932, 1 2018. doi:10.1016/j.jacep.2018.03.007

[81] B. M. Rocha, F. Kickinger, A. J. Prassl, G. Haase, E. J. Vigmond, R. W. Dos Santos, S. Zaglmayr, and G. Plank, "A macro finite-element formulation for cardiac electrophysiology simulations using hybrid unstructured grids," IEEE Trans. Biomed. Eng., vol. 58, no. 4, pp. 1055-1065, apr 2011. doi:10.1109/TBME.2010.2064167. http://ieeexplore.ieee.org/xpls/abs $\{$ "all.jsp?arnumber=5545378

[82] C. M. Augustin, A. Neic, M. Liebmann, A. J. Prassl, S. A. Niederer, G. Haase, and G. Plank, "Anatomically accurate high resolution modeling of human whole heart electromechanics: A strongly scalable algebraic multigrid solver method for nonlinear deformation." J. Comput. Phys., vol. 305, pp. 622-646, jan 2016. doi:10.1016/j.jcp.2015.10.045. http://www.ncbi.nlm.nih.gov/pubmed/26819483http: //www.pubmedcentral.nih.gov/articlerender.fcgi?artid=PMC4724941

[83] J. A. Southern, G. Plank, E. J. Vigmond, and J. P. Whiteley, "Solving the coupled system improves computational efficiency of the bidomain equations." IEEE Trans. Biomed. Eng., vol. 56, no. 10, pp. 2404-12, oct 2009. doi:10.1109/TBME.2009.2022548. http://www.ncbi.nlm.nih.gov/pubmed/19457741

[84] S. Abhyankar, J. Brown, E. M. Constantinescu, D. Ghosh, B. F. Smith, and H. Zhang, "PETSc/TS: A modern scalable ODE/DAE solver library," arXiv preprint arXiv:1806.01437, 2018. https://arxiv.org/abs/1806.01437

[85] R. Weber dos Santos, G. Plank, S. Bauer, and E. J. Vigmond, "Parallel multigrid preconditioner for the cardiac bidomain model." IEEE Trans. Biomed. Eng., vol. 51, no. 11, pp. 1960-8, nov 2004. doi:10.1109/TBME.2004.834275. http://www.ncbi.nlm.nih.gov/pubmed/15536898

[86] G. Plank, M. Liebmann, R. Weber dos Santos, E. J. Vigmond, and G. Haase, "Algebraic multigrid preconditioner for the cardiac bidomain model." IEEE Trans. Biomed. Eng., vol. 54, no. 4, pp. 585-96, apr 2007. doi:10.1109/TBME.2006.889181. http://ieeexplore.ieee.org/lpdocs/epic03/wrapper.htm?arnumber= 4132940http://www.ncbi.nlm.nih.gov/pubmed/17405366

[87] A. Neic, M. Liebmann, E. Hoetzl, L. Mitchell, E. J. Vigmond, G. Haase, and G. Plank, “Accelerating cardiac bidomain simulations using graphics processing units." IEEE Trans. Biomed. Eng., vol. 59, no. 8, pp. 2281-90, aug 2012. doi:10.1109/TBME.2012.2202661. http://www.ncbi.nlm.nih.gov/pubmed/22692867

[88] E. J. Vigmond, P. M. Boyle, L. Leon, and G. Plank, "Near-real-time simulations of biolelectric activity in small mammalian hearts using graphical processing units." Conf. Proc. ... Аnnu. Int. Conf. IEEE Eng. Med. Biol. Soc. IEEE Eng. Med. Biol. Soc. Annu. Conf., vol. 2009, pp. 3290-3, jan 2009. doi:10.1109/IEMBS.2009.5333738. http://www.pubmedcentral.nih.gov/articlerender.fcgi?artid=3977140 $\{\&\}$ tool $=$ pmcentrez $\{\&\}$ rendertype $=$ abstract 
[89] G. Plank, L. Zhou, J. L. Greenstein, S. Cortassa, R. L. Winslow, B. O'Rourke, and N. A. Trayanova, "From mitochondrial ion channels to arrhythmias in the heart: computational techniques to bridge the spatio-temporal scales." Philos. Trans. A. Math. Phys. Eng. Sci., vol. 366, no. 1879, pp. 3381-409, sep 2008. doi:10.1098/rsta.2008.0112. http://www.pubmedcentral.nih.gov/articlerender.fcgi? $\operatorname{artid}=2778066$ $\{\&\}$ tool=pmcentrez $\{\&\}$ rendertype $=$ abstract

[90] A. C. Hindmarsh, P. N. Brown, K. E. Grant, S. L. Lee, R. Serban, D. E. Shumaker, and C. S. Woodward, "Sundials: Suite of nonlinear and differential/algebraic equation solvers," ACM Trans. Math. Softw., vol. 31, no. 3, p. 363-396, Sep. 2005. doi:10.1145/1089014.1089020

[91] openCARP consortium, C. Augustin, J. Bayer et al., “opencarp (v5.0),” 2021. doi:10.35097/389

[92] A. Loewe, G. Seemann, E. M. Wülfers, Y.-L. Huang, J. Sanchez, F. Bach, R. Ulrich, and M. Selzer, "SuLMaSS - sustainable lifecycle management for scientific software," in E-Science-Tage 2019: Data to Knowledge, Heidelberg, 7 2019. doi:10.11588/heidok.00026843

[93] DataCite Metadata Working Group, "Datacite metadata schema documentation for the publication and citation of research data v4.3," 2019. doi:10.14454/7XQ3-ZF69

[94] U. Ayachit, The paraview guide: a parallel visualization application. Kitware, Inc., 2015.

[95] J. Bayer, A. J. Prassl, A. Pashaei, J. F. Gomez, A. Frontera, A. Neic, G. Plank, and E. J. Vigmond, "Universal ventricular coordinates: A generic framework for describing position within the heart and transferring data," Medical Image Analysis, vol. 45, pp. 83 - 93, 2018. doi:10.1016/j.media.2018.01.005

[96] C. H. Roney, A. Pashaei, M. Meo, R. Dubois, P. M. Boyle, N. A. Trayanova, H. Cochet, S. A. Niederer, and E. J. Vigmond, "Universal atrial coordinates applied to visualisation, registration and construction of patient specific meshes,” Med. Image Anal., vol. 55, pp. 65-75, 2019. doi:10.1016/j.media.2019.04.004

[97] S. Schuler, N. Pilia, D. Potyagaylo, and A. Loewe, "Cobiveco: Consistent biventricular coordinates for precise and intuitive description of position in the heart - with matlab implementation," 2021. https://arxiv.org/abs/2102.02898

[98] M. Courtemanche, R. J. Ramirez, and S. Nattel, "Ionic mechanisms underlying human atrial action potential properties: insights from a mathematical model," The American journal of physiology, vol. 275, no. 1 Pt 2 , pp. H301-21, 7 1998. doi:10.1152/ajpheart.1998.275.1.H301

[99] J. T. Koivumäki, T. Korhonen, and P. Tavi, "Impact of sarcoplasmic reticulum calcium release on calcium dynamics and action potential morphology in human atrial myocytes: a computational study." PLoS computational biology, vol. 7, no. 1, p. e1001067, 1 2011. doi:10.1371/journal.pcbi.1001067

[100] K. H. W. J. ten Tusscher and A. V. Panfilov, "Alternans and spiral breakup in a human ventricular tissue model," American Journal of Physiology. Heart and Circulatory Physiology, vol. 291, no. 3, pp. H1088-100, 1 2006. doi:10.1152/ajpheart.00109.2006

[101] T. O'Hara, L. Virag, A. Varro, and Y. Rudy, "Simulation of the undiseased human cardiac ventricular action potential: model formulation and experimental validation," PLoS Computational Biology, vol. 7, no. 5, p. e1002061, 1 2011. doi:10.1371/journal.pcbi.1002061

[102] A. Loewe, M. Wilhelms, O. Dössel, and G. Seemann, "Influence of chronic atrial fibrillation induced remodeling in a computational electrophysiological model," Biomedizinische Technik / Biomedical Engineering, vol. 59, no. S1, pp. S929-S932, 2014. doi:10.1515/bmt-2014-5012

[103] M. Clerx, P. Collins, E. de Lange, and P. G. A. Volders, "Myokit: A simple interface to cardiac cellular electrophysiology," Progress in Biophysics and Molecular Biology, vol. 120, no. 1-3, pp. 100-114, 12016. doi:10.1016/j.pbiomolbio.2015.12.008

[104] C. Mendonca Costa, E. Hoetzl, B. Martins Rocha, A. J. Prassl, and G. Plank, "Automatic parameterization strategy for cardiac electrophysiology simulations," Comput. Cardiol. (2010)., vol. 40, no. 2010, pp. 373$376,2013$.

[105] D. U. J. Keller, D. L. Weiss, O. Dössel, and G. Seemann, "Influence of I(Ks) heterogeneities on the genesis of the t-wave: a computational evaluation," IEEE Trans. Biomed. Engineering, vol. 59, pp. 311-322, 2012. doi:10.1109/TBME.2011.2168397

[106] S. A. Niederer, Y. Aboelkassem, C. D. Cantwell et al., "Creation and application of virtual patient cohorts of heart models: Virtual Cohorts of Heart Models," Philos. Trans. R. Soc. A Math. Phys. Eng. Sci., vol. 378, no. 2173, 2020. doi:10.1098/rsta.2019.0558 
bioRxiv preprint doi: https://doi.org/10.1101/2021.03.01.433036; this version posted June 10,2021 . The copyright holder for this preprint (which was not certified by peer review) is the author/funder, who has granted bioRxiv a license to display the preprint in perpetuity. It is made available under aCC-BY 4.0 International license.

[107] J. D. Bayer, R. C. Blake, G. Plank, and N. A. Trayanova, "A novel rule-based algorithm for assigning myocardial fiber orientation to computational heart models," Annals of Biomedical Engineering, vol. 40, no. 10, pp. 2243-2254, 1 2012. doi:10.1007/s10439-012-0593-5

[108] K. Gillette, J. Bouyssier, M. Gsell, A. Prassl, A. Neic, E. Vigmond, and G. Plank, "Automated Framework for the Inclusion of a Purkinje System in Cardiac Digital Twins of Ventricular Electrophysiology," Ann. Biomed. Eng., under review.

[109] K. Gillette, M. Gsell, A. Prassl et al., "A Framework for the Generation of Digital Twins of Cardiac Electrophysiology from Clinical 12-leads ECGs," Med. Imag. Anal., 2021. doi:10.1016/j.media.2021.102080

[110] S. Schuler and A. Loewe, "Biventricular statistical shape model of the human heart adapted for computer simulations," Jan. 2021. doi:10.5281/zenodo.4419784

[111] C. Nagel, S. Schuler, O. Dössel, and A. Loewe, "A Bi-atrial Statistical Shape Model and 100 Volumetric Anatomical Models of the Atria," Dec. 2020. doi:10.5281/zenodo.4309958

[112] S. Pezzuto, P. Kal'avský, M. Potse, F. W. Prinzen, A. Auricchio, and R. Krause, "Evaluation of a rapid anisotropic model for ECG simulation," Frontiers in Physiology, vol. 8, p. 265, May 2017. doi:10.3389/fphys.2017.00265

[113] T. E. Fastl, C. Tobon-Gomez, A. Crozier et al., "Personalized computational modeling of left atrial geometry and transmural myofiber architecture," Medical Image Analysis, vol. 47, pp. 180 - 190, 2018. doi:10.1016/j.media.2018.04.001

[114] A. Wachter, A. Loewe, M. W. Krueger, O. Dössel, and G. Seemann, "Mesh structure-independent modeling of patient-specific atrial fiber orientation," Current Directions in Biomedical Engineering, vol. 1, no. 1, pp. 409-412, 1 2015. doi:10.1515/cdbme-2015-0099

[115] A. Wachter and A. Loewe, "Resilient," 2021. doi:10.5281/ZENODO.4738369

[116] R. Piersanti, P. C. Africa, M. Fedele, C. Vergara, L. Dedè, A. F. Corno, and A. Quarteroni, "Modeling cardiac muscle fibers in ventricular and atrial electrophysiology simulations," Computer Methods in Applied Mechanics and Engineering, vol. 373, p. 113468, 1 2021. doi:10.1016/j.cma.2020.113468

[117] G. Seemann, C. Höper, F. B. Sachse, O. Dössel, A. V. Holden, and H. Zhang, "Heterogeneous threedimensional anatomical and electrophysiological model of human atria," Philos Trans A Math Phys Eng Sci., vol. 364, no. 1843, pp. 1465-1481, 1 2006. doi:10.1098/rsta.2006.1781

[118] C. Corrado, S. Williams, R. Karim, G. Plank, M. O'Neill, and S. Niederer, "A work flow to build and validate patient specific left atrium electrophysiology models from catheter measurements," Medical Image Analysis, vol. 47, pp. 153 - 163, 2018. doi:10.1016/j.media.2018.04.005

[119] P. M. Boyle, T. Zghaib, S. Zahid et al., "Computationally guided personalized targeted ablation of persistent atrial fibrillation.” Nature biomedical engineering, 8 2019. doi:10.1038/s41551-019-0437-9

[120] K. Gillette, A. Prassl, A. Neic, G. Plank, J. Bayer, and E. Vigmond, "Automatic generation of bi-ventricular models of cardiac electrophysiology for patient specific personalization using non-invasive recordings," in 2018 Computing in Cardiology Conference (CinC), vol. 45, 2018, pp. 1-4. doi:10.22489/CinC.2018.265

[121] W. Kahlmann, E. Poremba, D. Potyagaylo, O. Dössel, and A. Loewe, "Modelling of patient-specific purkinje activation based on measured ECGs," in Current Directions in Biomedical Engineering, vol. 3, no. 2. de Gruyter, 1 2017, pp. 171-174. doi:10.1515/cdbme-2017-0177

[122] C. H. Roney, A. Pashaei, M. Meo, R. Dubois, P. M. Boyle, N. A. Trayanova, H. Cochet, S. A. Niederer, and E. J. Vigmond, "Universal atrial coordinates applied to visualisation, registration and construction of patient specific meshes," Medical Image Analysis, vol. 55, pp. 65 - 75, 2019. doi:10.1016/j.media.2019.04.004

[123] L. Azzolin, S. Schuler, O. Dössel, and A. Loewe, "A reproducible protocol to assess arrhythmia vulnerability in silico: Pacing at the end of the effective refractory period," Frontiers in Physiology, pp. under review, medRxiv preprint available, 2021. doi:10.1101/2021.01.21.21250205

[124] M. J. Bishop and G. Plank, "Simulating photon scattering effects in structurally detailed ventricular models using a Monte Carlo approach," Front. Physiol., vol. 5, no. September, pp. 1-14, sep 2014. doi:10.3389/fphys.2014.00338

[125] _ - "Biophotonic modelling of cardiac optical imaging," vol. 859, pp. 367-404, 2015. doi:10.1007/9783-319-17641-3_15 
bioRxiv preprint doi: https://doi org/10.1101/2021.03.01 433036; this version posted June 10,2021 . The copyright holder for this preprint (which was not certified by peer review) is the author/funder, who has granted bioRxiv a license to display the preprint in perpetuity. It is made available under aCC-BY 4.0 International license.

[126] D. Frisch, T. G. Oesterlein, L. A. Unger, G. Lenis, R. Wakili, C. Schmitt, A. Luik, O. Doessel, and A. Loewe, "Mapping and removing the ventricular far field component in unipolar atrial electrograms." IEEE Transactions on Biomedical Engineering, vol. 67, no. 10, pp. 2905 -2915, 22020. doi:10.1109/TBME.2020.2973471

[127] M. J. Bishop, E. Vigmond, and G. Plank, "Cardiac bidomain bath-loading effects during arrhythmias: Interaction with anatomical heterogeneity," Biophys. J., vol. 101, no. 12, pp. 2871-2881, 2011. doi:10.1016/j.bpj.2011.10.052

[128] M. J. Bishop and G. Plank, "Bidomain ECG simulations using an augmented monodomain model for the cardiac source." IEEE transactions on bio-medical engineering, vol. 58, no. 8, 82011. doi:10.1109/TBME.2011.2148718

[129] — - "Representing cardiac bidomain bath-loading effects by an augmented monodomain approach: Application to complex ventricular models," IEEE Trans. Biomed. Eng., vol. 58, no. 4, pp. 1066-1075, 2011. doi:10.1109/TBME.2010.2096425

[130] A.-M. Plancke, A. Connolly, P. M. Gemmell et al., "Generation of a cohort of whole-torso cardiac models for assessing the utility of a novel computed shock vector efficiency metric for ICD optimisation." Comput. Biol. Med., vol. 112, p. 103368, 2019. doi:10.1016/j.compbiomed.2019.103368

[131] J. Sánchez, M. Nothstein, A. Neic et al., "openCARP: An open sustainable framework for in-silico cardiac electrophysiology research," in Computing in Cardiology Conference (CinC), ser. Computing in Cardiology Conference (CinC), vol. 47, 1 2020. doi:10.22489/CinC.2020.111

[132] M. D. Wilkinson, M. Dumontier, I. J. J. Aalbersberg et al., "The FAIR guiding principles for scientific data management and stewardship." Scientific data, vol. 3, p. 160018, 3 2016. doi:10.1038/sdata.2016.18

[133] F. Bach, K. Soltau, and M. Razum, "Bedarfsgerechte weiterentwicklung von radar als forschungsdatenrepositorium für das kit," in E-Science-Tage 2019: Data to Knowledge, V. Heuveline, F. Gebhart, and N. Mohammadianbisheh, Eds. University Library Heidelberg, 2019, pp. 162-172. doi:10.11588/HEIBOOKS.598

[134] B. F. Lavoie, "The open archival information system reference model: Introductory guide," Microform \& imaging review, vol. 33, no. 2, pp. 68-81, 2004. doi:10.1515/MFIR.2004.68

[135] H. Pampel, P. Vierkant, F. Scholze, R. Bertelmann, M. Kindling, J. Klump, H.-J. Goebelbecker, J. Gundlach, P. Schirmbacher, and U. Dierolf, "Making research data repositories visible: The re3data.org registry," PLOS ONE, vol. 8, no. 11, pp. 1-10, 11 2013. doi:10.1371/journal.pone.0078080 